\title{
Health Benefits of Integrating Physical Activity into Our Day-to-Day Lives
}

\author{
Rashmi Aggarwal' \\ ${ }^{1}$ Department of Thyroid and Endocrine Research, Institute of \\ Nuclear Medicine and Allied Sciences (INMAS), Timarpur, \\ Delhi, India
}

\begin{abstract}
Address for correspondence Rashmi Aggarwal, MD, Department of Thyroid and Endocrine Research, Institute of Nuclear Medicine and Allied Sciences-INMAS, Brig. S. K. Majumdar Marg, Timarpur, Delhi 110054, India (e-mail: drarashmi@yahoo.co.in).
\end{abstract}

\begin{abstract}
Keywords

- physical activity

- hypertension

- metabolic syndrome

- diabetes

In the 21st century it is the sedentary lifestyle that is responsible for causation of the dual epidemic of obesity and type 2 diabetes. Incorporating physical activity into our daily lives will not only improve our physical health but also enhance our mental and neurocognitive functions. Regular physical activity reduces the risks of developing metabolic derangements such as overweight, hypertension, different types of diabetes, metabolic syndrome, nonalcoholic fatty liver disease, osteoporosis, and certain types of organ-specific cancers.
\end{abstract}

It is now the right time we get serious about incorporating physical activity into our daily lives. Engaging in physical activity has innumerable benefits both on psychological and physical health. Persistent physical activity is associated with reduction in all-cause mortality plus cardiovascular mortality.

1. Physical activity also reduces the risk of developing a plethora of lifestyle diseases such as obesity, hypertension (HT), stroke, cancer, type 2 diabetes mellitus, and metabolic syndrome. Physical activity improves neurocognitive and physiologic functions. ${ }^{1}$

2. Today the major burden of noncommunicable diseases is attributable to sedentary lifestyle. Physical activity should form an integral part of disease prevention programs. People who regularly participate in optimal amounts of physical activity live longer, healthier, and happier lives.

\section{Diseases Associated with Physical Inactivity (Sedentary Lifestyle)}

Sedentary lifestyle plays an important role in pathogenesis of numerous chronic lifestyle disorders such as obesity, coronary artery disease, type 2 diabetes mellitus, depression, and certain type of cancers $^{2}$ (- Table $\mathbf{1}$ ).

\section{Physical Activity and Hypertension}

Hypertension (HT) is a major public health problem in India and is also an important risk factor for stroke, coronary artery disease, and chronic renal failure. Apart from other lifestyle modifications, physical activity remains the gold standard therapy both for prevention and treatment of HT. Huang et al studied the effect of aerobic exercise in elderly individuals with HT and found favorable results. ${ }^{3}$ In 2011, Cornelissen et al conducted a meta-analysis on the blood pressure (BP)-lowering effects of resistance training pooling data from 28 randomized controlled trials. Resistance training resulted in a net decrease of $3.9 \mathrm{~mm} \mathrm{Hg}$ and $3.9 \mathrm{~mm} \mathrm{Hg}$ in systolic and diastolic BP, respectively, in normotensive/prehypertensive participants. $^{4}$

\section{Physical Activity and Diabetes}

Physical activity has significant positive effects on blood glucose concentration in persons with both types 1 and 2 diabetes. ${ }^{2}$ Regular physical activity improves several parameters that are important in management of diabetes such as improving insulin sensitivity, controlling body mass, lowering lipid, and boosting the patient's self-esteem. ${ }^{2}$

\section{Physical Activity and Prevention of Cancer}

Bad lifestyle is a major risk factor for certain types of cancers such as cancer of the prostate, colon, breast, ovary, and endometrium.

Men and women who engage themselves in regular physical activity showed a 30 to $40 \%$ reduction in relative risk of colon cancer, and physically active women showed a 20 to
DOI https://doi.org/ $10.1055 / \mathrm{s}-0038-1675669$ ISSN 2321-0656.
License terms

() (1) $\Theta \circledast$ 
Table 1 Diseases related to sedentary lifestyle

\begin{tabular}{|l|}
\hline - Obesity \\
\hline - Prediabetes \\
\hline - Type 2 diabetes mellitus (DM) \\
\hline - Nonalcoholic fatty liver disease \\
\hline - Metabolic syndrome \\
\hline - Dyslipidemia \\
\hline - Hypertension (HT) \\
\hline - Osteoporosis \\
\hline - Cancer \\
\hline - Depression \\
\hline - Dementia
\end{tabular}

$30 \%$ reduction in relative risk of breast cancer compared with their counterparts who were inactive and sedentary. ${ }^{5}$

\section{Physical Activity and Osteoporosis}

Incorporating physical activity in our daily lives is important not only for preservation of bone health but also for prevention of osteopenia and osteoporosis, especially in postmenopausal women.

\section{Physical Activity and Sleep}

Reid et al noticed that aerobic physical activity with sleep hygiene education is an effective treatment modality to improve sleep in individuals with chronic insomnia. Engaging in physical activity not only improves sleep but also improves mood and overall quality of life in these individuals. ${ }^{6}$

\section{Probable Mechanism of Health Benefits due to Physical Activity}

Physical activity induces mitochondria biogenesis not only in skeletal muscles but also in other organs such as muscles, liver, brain, and kidney. ${ }^{7}$ This mitochondrial biogenesis probably is responsible for reduced all-cause mortality in physically active individuals.

\section{Conclusion}

Despite the overwhelming evidence of multiple health benefits of physical activity, most of the people across the globe are not sufficiently active to reap these health benefits of physical activity. Physical activity is for everyone, and any intensity of activity is better for our health than not involving ourselves in any activity at all and leading a sedentary life. Physical activity promotes cardiorespiratory fitness and improves bone health and cognitive functions. Regular physical activity plays an important role in prevention and treatment of many lifestyle-related chronic health diseases such as obesity, different types of diabetes (including prediabetes, type 1 and type 2 diabetes, gestational diabetes) HT, nonalcoholic fatty liver disease, dyslipidemia, metabolic syndrome, osteoporosis, and some organ-specific cancers. In view of enough clinical evidence that supports the dictum that regular physical activity is absolutely essential for maintenance of good health and overall well-being, it is high time that people across the globe and especially those residing in south Asian countries who are already facing the challenge of coping up with the dual epidemic of obesity (including childhood obesity) and type 2 diabetes must ensure integration of physical activity into their daily lives. Governmental and non-governmental organizations, district administration, school authorities, teachers, and parents should all encourage children to incorporate physical activity into their lifestyle.

\section{Conflict of Interest}

None.

\section{References}

1 Chaddock L, Erickson KI, Prakash RS, et al. A neuroimaging investigation of the association between aerobic fitness, hippocampal volume, and memory performance in preadolescent children. Brain Res 2010;1358:172-183

2 Chipkin SR, Klugh SA, Chasan-Taber L. Exercise and diabetes. Cardiol Clin 2001;19(3):489-505

3 Huang G, Shi X, Gibson CA, Huang SC, Coudret NA, Ehlman MC. Controlled aerobic exercise training reduces resting blood pressure in sedentary older adults. Blood Press 2013; 22(6):386-394

4 Cornelissen VA, Fagard RH, Coeckelberghs E, Vanhees L. Impact of resistance training on blood pressure and other cardiovascular risk factors: a meta-analysis of randomized, controlled trials. Hypertension 2011;58(5):950-958

5 Lee IM. Physical activity and cancer prevention-data from epidemiologic studies. Med Sci Sports Exerc 2003;35(11): 1823-1827

6 Reid KJ, Baron KG, Lu B, Naylor E, Wolfe L, Zee PC. Aerobic exercise improves self-reported sleep and quality of life in older adults with insomnia. Sleep Med 2010;11(9):934-940

7 Hood DA, Uguccioni G, Vainshtein A, D'souza D. Mechanisms of exercise-induced mitochondrial biogenesis in skeletal muscle: implications for health and disease. Compr Physiol 2011;1(3):1119-1134 